JOURNAL OF

SYMPLECTIC GEOMETRY

Volume 9, Number 4, 429-434, 2011

\title{
COHOMOLOGICALLY SYMPLECTIC SOLVMANIFOLDS ARE SYMPLECTIC
}

\author{
HisASHI KASUYA
}

\begin{abstract}
We consider aspherical manifolds with torsion-free virtually polycyclic fundamental groups, constructed by Baues. We prove that if those manifolds are cohomologically symplectic then they are symplectic. As a corollary we show that cohomologically symplectic solvmanifolds are symplectic.
\end{abstract}

\section{Introduction}

A $2 n$-dimensional compact manifold $M$ is called cohomologically symplectic (c-symplectic) if we have $\omega \in H^{2}(M, \mathbb{R})$ such that $\omega^{n} \neq 0$. A compact symplectic manifold is c-symplectic but the converse is not true in general. For example $\mathbb{C} P^{2} \# \mathbb{C} P^{2}$ is c-symplectic but not symplectic. But for some class of manifolds these two conditions are equivalent. For examples, nilmanifolds, i.e., compact homogeneous spaces of nilpotent simply connected Lie groups. In [7], for a nilpotent simply connected Lie group $G$ with a cocompact discrete subgroup $\Gamma$ (such subgroup is called a lattice), Nomizu showed that the de Rham cohomology $H^{*}(G / \Gamma, \mathbb{R})$ of $G / \Gamma$ is isomorphic to the cohomology $H^{*}(\mathfrak{g})$ of the Lie algebra of $G$. By the application of Nomizu's theorem, if $G / \Gamma$ is c-symplectic then $G / \Gamma$ is symplectic (see $[\mathbf{3}, \mathbf{p .} \mathbf{1 9 1}]$ ). Every nilmanifold can be represented by such $G / \Gamma$ (see $[6]$ ).

Consider solvmanifolds, i.e., compact homogeneous spaces of solvable simply connected Lie groups. Let $G$ be a solvable simply connected Lie group with a lattice $\Gamma$. We assume that for any $g \in G$ the all eigenvalues of the adjoint operator $\mathrm{Ad}_{g}$ are real. With this assumption, in [5] Hattori extended Nomizu's theorem. By Hattori's theorem, for such case, without difficulty, we can similarly show that if $G / \Gamma$ is c-symplectic, then $G / \Gamma$ is symplectic. But the isomorphism $H^{*}(G / \Gamma, \mathbb{R}) \cong H^{*}(\mathfrak{g})$ fails to hold for general solvable Lie groups, and not all solvmanifolds can be represented by $G / \Gamma$. Thus it is a considerable problem whether every c-symplectic solvmanifold is symplectic. 
Let $\Gamma$ be a torsion-free virtually polycyclic group. In [1] Baues constructed the compact aspherical manifold $M_{\Gamma}$ with $\pi_{1}\left(M_{\Gamma}\right)=\Gamma$. Baues proved that every infra-solvmanifold (see [1] for the definition) is diffeomorphic to $M_{\Gamma}$. In particular, the class of such aspherical manifolds contains the class of solvmanifolds. We prove that if $M_{\Gamma}$ is c-symplectic then $M_{\Gamma}$ is symplectic. In other words, for a torsion-free virtually polycyclic group $\Gamma$ with $2 n=\operatorname{rank} \Gamma$, if there exists $\omega \in H^{2}(\Gamma, \mathbb{R})$ such that $\omega^{n} \neq 0$ then we have a symplectic aspherical manifold with the fundamental group $\Gamma$.

\section{Notation and conventions}

A general reference here is [2]. Let $k$ be a subfield of $\mathbb{C}$. A group $\mathbf{G}$ is called a $k$-algebraic group if $\mathbf{G}$ is a Zariski-closed subgroup of $G L_{n}(\mathbb{C})$ which is defined by polynomials with coefficients in $k$. Let $\mathbf{G}(k)$ denote the set of $k$-points of $\mathbf{G}$ and $\mathbf{U}(\mathbf{G})$ the maximal Zariski-closed unipotent normal $k$ subgroup of $\mathbf{G}$ called the unipotent radical of $\mathbf{G}$. Let $U_{n}(k)$ denote the $n \times n$ $k$-valued upper triangular unipotent matrix group.

\section{Aspherical manifolds with torsion-free virtually polycyclic fundamental groups}

Definition 3.1. A group $\Gamma$ is polycyclic if it admits a sequence

$$
\Gamma=\Gamma_{0} \supset \Gamma_{1} \supset \cdots \supset \Gamma_{k}=\{e\}
$$

of subgroups such that each $\Gamma_{i}$ is normal in $\Gamma_{i-1}$ and $\Gamma_{i-1} / \Gamma_{i}$ is cyclic. We denote $\operatorname{rank} \Gamma=\sum_{i=1}^{i=k} \operatorname{rank} \Gamma_{i-1} / \Gamma_{i}$.

Proposition 3.1 [8, Proposition 3.10]. The fundamental group of a solvmanifold is torsion-free polycyclic.

Let $k$ be a subfield of $\mathbb{C}$. Let $\Gamma$ be a torsion-free virtually polycyclic group. For a finite index polycyclic subgroup $\Delta \subset \Gamma$, we denote $\operatorname{rank} \Gamma=\operatorname{rank} \Delta$.

Definition 3.2. We call a k-algebraic group $\boldsymbol{H}_{\Gamma}$ a $k$-algebraic hull of $\Gamma$ if there exists an injective group homomorphism $\psi: \Gamma \rightarrow \boldsymbol{H}_{\Gamma}(k)$ and $\boldsymbol{H}_{\Gamma}$ satisfies the following conditions:

(1) $\psi(\Gamma)$ is Zariski-dense in $\mathbf{H}_{\boldsymbol{\Gamma}}$.

(2) $Z_{\boldsymbol{H}_{\Gamma}}\left(\boldsymbol{U}\left(\boldsymbol{H}_{\Gamma}\right)\right) \subset \boldsymbol{U}\left(\boldsymbol{H}_{\Gamma}\right)$ where $Z_{\boldsymbol{H}_{\Gamma}}\left(\boldsymbol{U}_{(}\left(\boldsymbol{H}_{\Gamma}\right)\right)$ is the centralizer of $\boldsymbol{U}\left(\boldsymbol{H}_{\Gamma}\right)$.

(3) $\operatorname{dim} \boldsymbol{U}\left(\boldsymbol{H}_{\Gamma}\right)=\operatorname{rank} \Gamma$.

Theorem 3.1 [1, Theorem A.1]. There exists a $k$-algebraic hull of $\Gamma$ and a $k$-algebraic hull of $\Gamma$ is unique up to $k$-algebraic group isomorphism.

Let $\Gamma$ be a torsion-free virtually polycyclic group and $\mathbf{H}_{\Gamma}$ the $\mathbb{Q}$-algebraic hull of $\Gamma$. Denote $H_{\Gamma}=\mathbf{H}_{\Gamma}(\mathbb{R})$. Let $U_{\Gamma}$ be the unipotent radical of $H_{\Gamma}$ 
and $T$ a maximal reductive subgroup. Then $H_{\Gamma}$ decomposes as a semi-direct product $H_{\Gamma}=T \ltimes U_{\Gamma}$. Let $\mathfrak{u}$ be the Lie algebra of $U_{\Gamma}$. Since the exponential map $\exp : \mathfrak{u} \longrightarrow U_{\Gamma}$ is a diffeomorphism, $U_{\Gamma}$ is diffeomorphic to $\mathbb{R}^{n}$ such that $n=\operatorname{rank} \Gamma$. For the semi-direct product $H_{\Gamma}=T \ltimes U_{\Gamma}$, we denote $\phi: T \rightarrow \operatorname{Aut}\left(U_{\Gamma}\right)$ the action of $T$ on $U_{\Gamma}$. Then we have the homomorphism $\alpha: H_{\Gamma} \longrightarrow \operatorname{Aut}\left(U_{\Gamma}\right) \ltimes U_{\Gamma}$ such that $\alpha(t, u)=(\phi(t), u)$ for $(t, u) \in T \ltimes U_{\Gamma}$. By the property (2) in Definition 3.2, $\phi$ is injective and hence $\alpha$ is injective.

In [1] Baues constructed a compact aspherical manifold $M_{\Gamma}=\alpha(\Gamma) \backslash U_{\Gamma}$ with $\pi_{1}\left(M_{\Gamma}\right)=\Gamma$. We call $M_{\Gamma}$ a standard $\Gamma$-manifold.

Theorem $3.2[\mathbf{1}$, Theorem 1.2, 1.4]. A standard $\Gamma$-manifold is unique up to diffeomorphism. A solvmanifold with the fundamental group $\Gamma$ is diffeomorphic to the standard $\Gamma$-manifold $M_{\Gamma}$.

Let $A^{*}\left(M_{\Gamma}\right)$ be the de Rham complex of $M_{\Gamma}$. Then $A^{*}\left(M_{\Gamma}\right)$ is the set of the $\Gamma$-invariant differential forms $A^{*}\left(U_{\Gamma}\right)^{\Gamma}$ on $U_{\Gamma}$. Let $\left(\bigwedge \mathfrak{u}^{*}\right)^{T}$ be the leftinvariant forms on $U_{\Gamma}$ which are fixed by $T$. Since $\Gamma \subset H_{\Gamma}=T \ltimes U_{\Gamma}$, we have the inclusion

$$
\left(\bigwedge \mathfrak{u}^{*}\right)^{T}=A^{*}\left(U_{\Gamma}\right)^{H_{\Gamma}} \subset A^{*}\left(U_{\Gamma}\right)^{\Gamma}=A^{*}\left(M_{\Gamma}\right) .
$$

Theorem 3.3 [1, Theorem 1.8]. This inclusion induces an isomorphism on cohomology.

By the application of the above facts, we prove the main theorem of this paper.

Theorem 3.4. Suppose $M_{\Gamma}$ is c-symplectic. Then $M_{\Gamma}$ admits a symplectic structure. In particular, cohomologically symplectic solvmanifolds are symplectic.

Proof. Since we have the isomorphism $H^{*}\left(M_{\Gamma}, \mathbb{R}\right) \cong H^{*}\left(\left(\bigwedge \mathfrak{u}^{*}\right)^{T}\right)$, we have $\omega \in\left(\bigwedge^{2} \mathfrak{u}^{*}\right)^{T}$ such that $0 \neq[\omega]^{n} \in H^{2 n}\left(\left(\bigwedge \mathfrak{u}^{*}\right)^{T}\right)$. This gives $0 \neq \omega^{n} \in$ $\left(\bigwedge \mathfrak{u}^{*}\right)^{T}$ and hence $0 \neq \omega^{n} \in \bigwedge \mathfrak{u}^{*}$. Since $\omega^{n}$ is a non-zero invariant $2 n$-form on $U_{\Gamma}$, we have $\left(\omega^{n}\right)_{p} \neq 0$ for any $p \in U_{\Gamma}$. Hence by the inclusion $\left(\bigwedge \mathfrak{u}^{*}\right)^{T} \subset$ $A^{*}\left(U_{\Gamma}\right)^{T}=A^{*}\left(M_{\Gamma}\right)$, we have $\left(\omega^{n}\right)_{\Gamma p} \neq 0$ for any $\Gamma p \in \Gamma \backslash U_{\Gamma}=M_{\Gamma}$. This implies that $\omega$ is a symplectic form on $M_{\Gamma}$. Hence, we have the theorem.

\section{Remarks}

Let $G=\mathbb{R} \ltimes_{\phi} U_{3}(\mathbb{C})$ such that

$$
\phi(t) \cdot\left(\begin{array}{ccc}
1 & x & z \\
0 & 1 & y \\
0 & 0 & 1
\end{array}\right)=\left(\begin{array}{ccc}
1 & \mathrm{e}^{\mathrm{i} \pi t} \cdot x & z \\
0 & 1 & \mathrm{e}^{-\mathrm{i} \pi t} \cdot y \\
0 & 0 & 1
\end{array}\right)
$$


and $D=\mathbb{Z} \ltimes_{\phi} D^{\prime}$ with

$$
D^{\prime}=\left\{\left(\begin{array}{ccc}
1 & x_{1}+\mathrm{i} x_{2} & z_{1}+\mathrm{i} z_{2} \\
0 & 1 & y_{1}+\mathrm{i} y_{2} \\
0 & 0 & 1
\end{array}\right): x_{1}, y_{2}, z_{2} \in \mathbb{Z}, x_{2}, y_{1}, z_{1} \in \mathbb{R}\right\}
$$

Then $D$ is not discrete and $G / D$ is compact. We have $D / D_{0} \cong \mathbb{Z} \ltimes_{\varphi} U_{3}(\mathbb{Z})$ such that

$$
\varphi(t) \cdot\left(\begin{array}{ccc}
1 & x & z \\
0 & 1 & y \\
0 & 0 & 1
\end{array}\right)=\left(\begin{array}{ccc}
1 & (-1)^{t} x & z \\
0 & 1 & (-1)^{-t} y \\
0 & 0 & 1
\end{array}\right)
$$

where $D_{0}$ is the identity component of $D$. Denote $\Gamma=D / D_{0}$. We have the algebraic hull $H_{\Gamma}=\{ \pm 1\} \ltimes_{\psi}\left(U_{3}(\mathbb{R}) \times \mathbb{R}\right)$ such that

$$
\psi(-1) \cdot\left(\left(\begin{array}{lll}
1 & x & z \\
0 & 1 & y \\
0 & 0 & 1
\end{array}\right), t\right)=\left(\left(\begin{array}{ccc}
1 & -x & z \\
0 & 1 & -y \\
0 & 0 & 1
\end{array}\right), t\right) .
$$

The dual of the Lie algebra $\mathfrak{u}$ of $U_{3}(\mathbb{R}) \times \mathbb{R}$ is given by $\mathfrak{u}^{*}=\langle\alpha, \beta, \gamma, \delta\rangle$ such that the differential is given by

$$
\begin{gathered}
d \alpha=d \beta=d \delta=0, \\
d \gamma=-\alpha \wedge \beta,
\end{gathered}
$$

and the action of $\{ \pm 1\}$ is given by

$$
\begin{gathered}
(-1) \cdot \alpha=-\alpha,(-1) \cdot \beta=-\beta, \\
(-1) \cdot \gamma=\gamma,(-1) \cdot \delta=\delta .
\end{gathered}
$$

Then we have a diffeomorphism $M_{\Gamma} \cong G / D$ and an isomorphism $H^{*}\left(M_{\Gamma}, \mathbb{R}\right) \cong H^{*}\left(\left(\bigwedge \mathfrak{u}^{*}\right)^{\{ \pm 1\}}\right)$. By simple computations, $H^{2}\left(\left(\bigwedge \mathfrak{u}^{*}\right)^{\{ \pm 1\}}\right)=0$ and hence the solvmanifold $G / D$ is not symplectic.

Remark 1. The proof of the Theorem 3.4 contains a proof of the following proposition.

Proposition 4.1. If $M_{\Gamma}$ admits a symplectic structure, then $U_{\Gamma}$ has an invariant symplectic form.

Otherwise for the above example, $U_{\Gamma}=U_{3}(\mathbb{R}) \times \mathbb{R}$ has an invariant symplectic form but $M_{\Gamma}$ is not symplectic. Thus the converse of this proposition is not true. If $\Gamma$ is nilpotent, then $T$ is trivial and any invariant symplectic form on $U_{\Gamma}$ induces the symplectic form on $M_{\Gamma}$. Hence for nilmanifolds, the converse of Proposition 4.1 is true.

Remark 2. $\Gamma$ is a finite extension of a lattice of $U_{\Gamma}=U_{3}(\mathbb{R}) \times \mathbb{R}$. Hence $M_{\Gamma}$ is finitely covered by a Kodaira-Thurston manifold (see $[\mathbf{9}],[\mathbf{3}, \mathbf{p .} \mathbf{1 9 2}]) \cdot M_{\Gamma}$ is an example of a non-symplectic manifold finitely covered by a symplectic manifold. 
Let $H=G \times \mathbb{R}$. Then the dual of the Lie algebra $\mathfrak{h}$ of $H$ is given by $\mathfrak{h}^{*}=\left\langle\sigma, \tau, \zeta_{1}, \zeta_{2}, \eta_{1}, \eta_{2}, \theta_{1}, \theta_{2}\right\rangle$ such that the differential is given by

$$
\begin{gathered}
d \sigma=d \tau=0, \\
d \zeta_{1}=\tau \wedge \zeta_{2}, d \zeta_{2}=-\tau \wedge \zeta_{1}, \\
d \eta_{1}=\tau \wedge \eta_{2}, d \eta_{2}=-\tau \wedge \eta_{1}, \\
d \theta_{1}=-\zeta_{1} \wedge \eta_{1}+\zeta_{2} \wedge \eta_{2}, d \theta_{2}=-\zeta_{1} \wedge \eta_{2}-\zeta_{2} \wedge \eta_{1} .
\end{gathered}
$$

By simple computations, any closed invariant 2-form $\omega \in \bigwedge^{2} \mathfrak{h}^{*}$ satisfies $\omega^{4}=0$. Hence $H$ has no invariant symplectic form. Otherwise we have a lattice $\Delta=2 \mathbb{Z} \ltimes U_{3}(\mathbb{Z}+i \mathbb{Z}) \times \mathbb{Z}$ which is also a lattice of $\mathbb{R}^{2} \times U_{3}(\mathbb{C})$. Thus $H / \Delta$ is diffeomorphic to a direct product of a two-dimensional torus and an Iwasawa manifold (see [4]). Since an Iwasawa manifold is symplectic (see $[4]), H / \Delta$ is also symplectic. By this example we can say:

Remark 3. For a simply connected nilpotent Lie group $G$ with a lattice $\Gamma$, if the nilmanifold $G / \Gamma$ is symplectic then $G$ has an invariant symplectic form. But suppose $G$ is solvable we have an example of a symplectic solvmanifold $G / \Gamma$ such that $G$ has no invariant symplectic form.

\section{References}

[1] O. Baues, Infra-solvmanifolds and rigidity of subgroups in solvable linear algebraic groups. Topology 43(4) (2004), 903-924.

[2] A. Borel, Linear Algebraic Groups 2nd enl. ed Springer-Verlag, 1991.

[3] Y. Félix, J. Oprea and D. Tanré, Algebraic Models in Geometry, Oxford Graduate Texts in Mathematics 17, Oxford University Press, 2008.

[4] M. Fernandez and A. Gray, The Iwasawa manifold. in Differential Geometry, Peniscola 1985, pp. 157-159, Lecture Notes in Mathematics, 1209, Springer, Berlin, 1986.

[5] A. Hattori, Spectral sequence in the de Rham cohomology of fibre bundles. J. Fac. Sci. Univ. Tokyo Sect. I 8 (1960), 289-331.

[6] A. Malcev, On a class of homogeneous spaces. (in Russian) Izvestiya Akad. Nauk. SSSR. Ser. Mat. 13 (1949), 9-32.

[7] K. Nomizu, On the cohomology of compact homogeneous spaces of nilpotent Lie groups. Ann. Math. 59(2) (1954), 531-538.

[8] M. S. Raghunathan, Discrete Subgroups of Lie Groups, Springer-Verlag, New York, 1972.

[9] W. P. Thurston, Some simple examples of symplectic manifolds. Proc. Amer. Math. Soc. 55(2) (1976), 467-468.

Graduate School of Mathematical Science

UNIVERSITY OF TOKYO

JAPAN

E-mail address: khsc@ms.u-tokyo.ac.jp

Received 07/30/2010, accepted 02/17/2011 
The author would like to express his gratitude to Toshitake Kohno for helpful suggestions and stimulating discussions. This research is supported by JSPS Research Fellowships for Young Scientists. 